\title{
Eigenvector Algorithms Using Reference Signals for Blind Source Separation of Instantaneous Mixtures
}

\author{
Mitsuru Kawamoto* $^{* \dagger}$, Kiyotaka Kohno ${ }^{\ddagger \uparrow}$, and Yujiro Inouye ${ }^{\ddagger \S}$ \\ * National Institute of Advanced Industrial Science and Technology (AIST) \\ Central 2, 1-1-1 Umezono, Tsukuba, Ibaraki 305-8568, Japan †'Email: m.kawamoto@aist.go.jp \\ BMC Research Center, RIKEN, Nagoya, 463-0003 Japan \\ ${ }_{\ddagger}^{\ddagger}$ Department of Electronic and Control Systems Engineering, Shimane University \\ 1060 Nishikawatsu, Matsue, Shimane 690-8504 Japan \\ IEmail: kohno@yonago-k.ac.jp, ${ }^{\S}$ Email: inouye@ riko.shimane-u.ac.jp
}

\begin{abstract}
This paper presents an eigenvector algorithm (EVA) derived from a criterion using reference signals, in which the EVA is applied to the blind source separation (BSS) of instantaneous mixtures. The proposed EVA works such that source signals are simultaneously separated from their mixtures. This is a new result, which has not been clarified by the conventional researches. Moreover, by modifying the criterion, the corresponding EVA which is robust to Gaussian noise is derived. Simulation results show the validity of the proposed EVAs.
\end{abstract}

\section{INTRODUCTION}

This paper deals with the blind source separation (BSS) problem for a multiple-input and multiple-output (MIMO) static system driven by independent source signals. To solve this problem, reference signals are used. Researches on the BSS problem by using the idea of the reference signal, to our best knowledge, have been made by Jelonnek et al. [4], [5] and Adib et al. [2]. Jelonnek et al. have proposed an eigenvector algorithm (EVA) derived from a criterion using reference signals, in order to solve blind equalization of single-input and single-output (SISO) systems. Adib et al. have proposed contrast functions for solving the BSS problem, in which reference signals are included into the contrast functions, but they have not derived explicit algorithms for solving the BSS problem from the contrast functions.

In this paper, the EVA derived from a criterion with reference signals is used for solving the BSS problem of MIMO static systems, and then it will be shown that the EVA works such that source signals are simultaneously separated from their mixtures. Moreover, by modifying the criterion, it will be shown that the EVA derived from the modified criterion consists of only higher-order cumulants and is able to work such that even if Gaussian noises are added to the outputs of the system, it can be used to achieve the BSS with as little the influence of Gaussian noise as possible. Simulation results show that the proposed EVAs can successfully solve the BSS problem.

\section{PROBLEM FORMULATION}

Throughout this paper, let us consider the following MIMO static system with $n$ inputs and $m$ outputs:

$$
\boldsymbol{y}(t)=\boldsymbol{H} \boldsymbol{s}(t)+\boldsymbol{n}(t),
$$

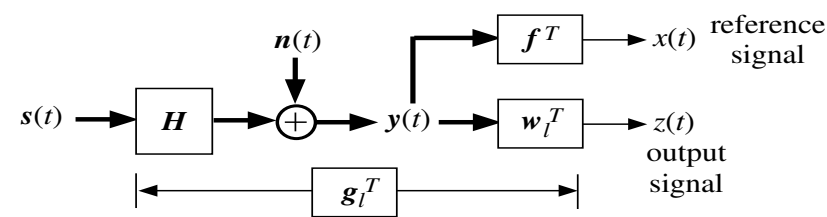

Fig. 1. The composite system of an unknown system and a filter, and reference system.

where $\boldsymbol{y}(t)$ represents an $m$-column output vector called the observed signal, $\boldsymbol{s}(t)$ represents an $n$-column input vector called the source signal, $\boldsymbol{H}$ is an $m \times n$ matrix, $\boldsymbol{n}(t)$ represents an $m$-column noise vector. It can be regarded as a linear mixture model with additive noise.

To achieve the blind source separation (BSS) for the system (1), the following $n$ filters, which are $m$-input single-output (MISO) static systems driven by the observed signals, are used:

$$
z_{l}(t)=\boldsymbol{w}_{l}^{T} \boldsymbol{y}(t), \quad l=1,2, \cdots, n,
$$

where $z_{l}(t)$ is the $l$ th output of the filter, $\boldsymbol{w}_{l}=$ $\left[w_{l 1}, w_{l 2}, \cdots, w_{l m}\right]^{T}$ is an $m$-column vector representing the $m$ coefficients of the filter. Substituting (1) into (2), we obtain

$$
\begin{aligned}
z_{l}(t) & =\boldsymbol{w}_{l}^{T} \boldsymbol{H} \boldsymbol{s}(t)+\boldsymbol{w}_{l}^{T} \boldsymbol{n}(t) \\
& =\boldsymbol{g}_{l}^{T} \boldsymbol{s}(t)+\boldsymbol{w}_{l}^{T} \boldsymbol{n}(t), \quad l=1,2, \cdots, n,
\end{aligned}
$$

where $\boldsymbol{g}_{l}=\left[g_{l 1}, g_{l 2}, \cdots, g_{l n}\right]^{T}:=\boldsymbol{H}^{T} \boldsymbol{w}_{l}$ is an $n$-column vector. The BSS problem considered in this paper can be formulated as follows: Find $n$ filters $\boldsymbol{w}_{l}$ 's denoted by $\tilde{\boldsymbol{w}}_{l}$ 's satisfying the following condition, without the knowledge of $\boldsymbol{H}$.

$$
\tilde{\boldsymbol{g}}_{l}=\boldsymbol{H}^{T} \tilde{\boldsymbol{w}}_{l}=\tilde{\boldsymbol{\delta}}_{l}, \quad l=1,2, \cdots, n,
$$

where $\tilde{\boldsymbol{\delta}}_{l}$ is an $n$-column vector whose elements $\tilde{\delta}_{l r}(r=$ $1,2, \cdots, n)$ are equal to zero expect for $\rho_{l}$ th element, that is, $\tilde{\delta}_{l r}=c_{l} \delta\left(r-\rho_{l}\right), r=1,2, \cdots, n$. Here, $\delta(t)$ is the Kronecker delta function, $c_{l}$ is a number standing for a scale change, and $\rho_{l}$ is one of integers $\{1,2, \cdots, n\}$ such that the set $\left\{\rho_{1}, \rho_{2}, \cdots, \rho_{n}\right\}$ is a permutation of the set $\{1,2, \cdots, n\}$.

To solve the BSS problem, we put the following assumptions on the system and the source signals.

A1) The matrix $\boldsymbol{H}$ in (1) is an $m \times n(m \geq n)$ matrix and has full column rank. 
A2) The input sequence $\{\boldsymbol{s}(t)\}$ is a zero-mean, nonGaussian vector stationary process whose element processes $\left\{s_{i}(t)\right\}, i=1,2, \cdots, n$, are mutually statistically independent and have nonzero variance, $\sigma_{s_{i}}^{2}=E\left[s_{i}^{2}(t)\right] \neq 0$ and nonzero fourth-order cumulants, $\gamma_{i}$ defined as

$\gamma_{i}=\operatorname{cum}\left\{s_{i}(t), s_{i}(t), s_{i}(t), s_{i}(t)\right\} \neq 0$ for $i=1,2, \cdots, n$,

A3) The noise signal sequence $\{\boldsymbol{n}(t)\}$ is a zero-mean, Gaussian vector stationary process whose element processes $\left\{n_{i}(t)\right\}, i=1,2, \cdots, m$, are mutually statistically independent.

A4) The two vector sequences $\{\boldsymbol{n}(t)\}$ and $\{\boldsymbol{s}(t)\}$ are mutually statistically independent.

It is assumed for the sake of simplicity in this paper that all the signals and all the systems are real-valued.

\section{EIGENVECTOR ALGORITHMS (EVAS)}

\section{A. Analysis of eigenvector algorithms with reference signals} for MIMO static systems

In this subsection, we assume that there is no noise $\boldsymbol{n}(t)$ in the output $\boldsymbol{y}(t)$, and then analyze eigenvector algorithms for MIMO static systems. Under this assumption, to solve the BSS problem, the following cross-cumulant between $z_{l}(t)$ and a reference signal $x(t)$ (see Fig. 1) is defined:

$$
C_{z x}=\operatorname{cum}\left\{z_{l}(t), z_{l}(t), x(t), x(t)\right\}
$$

where the reference signal $x(t)$ is given by $\boldsymbol{f}^{T} \boldsymbol{y}(t)=$ $\boldsymbol{f}^{T} \boldsymbol{H} \boldsymbol{s}(t)=\boldsymbol{a}^{T} \boldsymbol{s}(t)\left(\boldsymbol{a}^{T}:=\boldsymbol{f}^{T} \boldsymbol{H}\right.$ is a vector whose elements are $\left.a_{1}, a_{2}, \cdots, a_{n}\right)$, using an appropriate filter $\boldsymbol{f}$. The filter $\boldsymbol{f}$ is called a reference system. Moreover we define the constraint $\sigma_{z_{l}}^{2}=\sigma_{s_{\rho_{l}}}^{2}$, where $\sigma_{z_{l}}^{2}$ and $\sigma_{s_{\rho_{l}}}^{2}$ denote the variances of the output $z_{l}(t)$ and a source signal $s_{\rho_{l}}(t)$, respectively. Adib, et al. [2] have shown that the BSS can be achieved by maximizing $\left|C_{z x}\right|$ in (6) under the constraint, but they have not proposed any algorithm for achieving this idea. In the single-input case, Jelonnek et al. [4], [5] have shown that by the Lagrangian method, the maximization of $\left|C_{z x}\right|$ under $\sigma_{z_{l}}^{2}=\sigma_{s_{\rho_{l}}}^{2}$ leads to a closed-form expression as the following generalized eigenvector problem:

$$
\boldsymbol{C}_{y x} \boldsymbol{w}_{l}=\lambda \boldsymbol{R} \boldsymbol{w}_{l}
$$

Then they utilize the facts that $C_{z x}$ and $\sigma_{z_{l}}^{2}$ can be expressed in terms of the vector $\boldsymbol{w}_{l}$ as, respectively,

$$
\begin{aligned}
& C_{z x}=\boldsymbol{w}_{l}^{T} \boldsymbol{C}_{y x} \boldsymbol{w}_{l}, \\
& \sigma_{z_{l}}^{2}=\boldsymbol{w}_{l}^{T} \boldsymbol{R} \boldsymbol{w}_{l},
\end{aligned}
$$

where $\boldsymbol{C}_{y x}$ is a matrix whose $(i, j)$ element is calculated by $\operatorname{cum}\left\{y_{i}(t), y_{j}(t), x(t), x(t)\right\}$ and $\boldsymbol{R}=E\left[\boldsymbol{y}(t) \boldsymbol{y}^{T}(t)\right]$ is the covariance matrix of $m$-column vector $\boldsymbol{y}(t)$. Moreover, they have shown that the eigenvector corresponding to the maximum eigenvalue of $\boldsymbol{R}^{\dagger} \boldsymbol{C}_{y x}$ becomes the solution of the blind equalization problem in [4], [5], which is referred to as an eigenvector algorithm (EVA). However, the algorithm proposed by Jelonnek et al. is for SISO or SIMO infinite impulse response channel. Therefore, we want to show how the eigenvector algorithm (7) works for the BSS in the case of the MIMO static system. To this end, we use the following equalities:

$$
\begin{gathered}
\boldsymbol{R}=\boldsymbol{H} \boldsymbol{\Sigma} \boldsymbol{H}^{T}, \\
\boldsymbol{C}_{y x}=\boldsymbol{H} \boldsymbol{\Lambda} \boldsymbol{H}^{T},
\end{gathered}
$$

(5) where $\boldsymbol{\Sigma}$ is a diagonal matrix whose elements are $\sigma_{s_{i}}^{2}, i=$ $1,2, \cdots, n$ and $\boldsymbol{\Lambda}$ is a diagonal matrix whose elements are $a_{i}^{2} \gamma_{i}$ $(i=1,2, \cdots, n)$. Then we obtain the following theorem.

Theorem 1: Suppose the values $a_{i}^{2} \gamma_{i} / \sigma_{s_{i}}^{2}, i=1,2, \cdots, n$ are all nonzero and distinct. If the noise $\boldsymbol{n}(t)$ is absent in (1), then the $n$ eigenvectors corresponding to $n$ nonzero eigenvalues of $\boldsymbol{R}^{\dagger} \boldsymbol{C}_{y x}$ become the vectors $\tilde{\boldsymbol{w}}_{l}^{\prime}$ 's satisfying (4), where the symbol $\dagger$ denotes the pseudo-inverse operation of a matrix.

Proof: Based on (7), we consider the following eigenvector problem:

$$
\boldsymbol{R}^{\dagger} \boldsymbol{C}_{y x} \boldsymbol{w}_{l}=\lambda \boldsymbol{w}_{l}
$$

Then, from (10) and (11), (12) becomes

$$
\boldsymbol{H}^{T \dagger} \boldsymbol{\Sigma}^{-1} \boldsymbol{H}^{\dagger} \boldsymbol{H} \boldsymbol{\Lambda} \boldsymbol{H}^{T} \boldsymbol{w}_{l}=\lambda \boldsymbol{w}_{l} .
$$

Since $\boldsymbol{H}$ has full column rank, using a property of the pseudoinverse operation ([7], p. 433), we obtain

$$
\boldsymbol{H}^{T \dagger} \boldsymbol{\Sigma}^{-1} \boldsymbol{\Lambda} \boldsymbol{H}^{T} \boldsymbol{w}_{l}=\lambda \boldsymbol{w}_{l} .
$$

Multiplying (14) by $\boldsymbol{H}^{T}$ from left side and using a property of the pseudo-inverse operation again, (14) becomes

$$
\boldsymbol{\Sigma}^{-1} \boldsymbol{\Lambda} \boldsymbol{H}^{T} \boldsymbol{w}_{l}=\lambda \boldsymbol{H}^{T} \boldsymbol{w}_{l}
$$

By noting that $\boldsymbol{\Sigma}^{-1} \boldsymbol{\Lambda}$ is a diagonal matrix whose elements, $a_{i}^{2} \gamma_{i} / \sigma_{s_{i}}^{2}, i=1,2, \cdots, n$, are all nonzero and distinct, if $\boldsymbol{g}_{l}$ $:=\boldsymbol{H}^{T} \boldsymbol{w}_{l} \neq 0$, then the eigenvectors $\boldsymbol{g}_{l}$ obtained from (15) become the vectors $\tilde{\boldsymbol{g}}_{l}$ satisfying (4). Namely, the $n$ eigenvectors $\boldsymbol{w}_{l}$ corresponding to $n$ nonzero eigenvalues of $\boldsymbol{R}^{\dagger} \boldsymbol{C}_{y x}$ obtained from (12) become the vectors $\tilde{\boldsymbol{w}}_{l}$ satisfying (4).

Remark 1: In order to use Theorem 1, the reference signal $x(t)$ contains nonzero contributions $a_{i}{ }^{\prime} \mathrm{s}$ from all source signals $s_{i}(t)^{\prime}$ s. This is the case except for pathological cases. From Theorem 1, it can be seen that by all the $n$ eigenvectors corresponding to $n$ nonzero eigenvalues of $\boldsymbol{R}^{\dagger} \boldsymbol{C}_{y x}$, all source signals can be separated from the output $\boldsymbol{y}(t)$. This is a novel result which has not been shown in the conventional researches. Moreover, it can be seen from (15) that even if the fourth-order cumulants $\gamma_{i}$ have different signs from each other, the vector $\tilde{\boldsymbol{w}}_{l}$ satisfying (4) can be obtained. This fact will be confirmed by computer simulations in Section V.

\section{B. Robust Eigenvector algorithm}

In the previous subsection, we assume that there are no noises in the output signals. In this subsection, we shall show such an eigenvector algorithm that the solutions (4) can be obtained, even if the noise $\boldsymbol{n}(t)$ is presented in the output $\boldsymbol{y}(t)$. To this end, we introduce fourth-order cumulants matrices of $m$-vector random process $\{\boldsymbol{y}(t)\}$ [9], which constitute a set of 
$m \times m$ matrices $\boldsymbol{C}_{\boldsymbol{y}, i}^{(4)}(i=1,2, \cdots, m)$. The $(q, r)$ th element of the matrix $C_{\boldsymbol{y}, i}^{(4)}$ is defined by

$$
\left[\boldsymbol{C}_{\boldsymbol{y}, i}^{(4)}\right]_{q, r}=\operatorname{cum}\left\{y_{q}(t), y_{r}(t), y_{i}(t), y_{i}(t)\right\},
$$

where $[\cdot]_{q, r}$ denotes the $(q, r)$ th element of the matrix $\boldsymbol{C}_{\boldsymbol{y}, i}^{(4)}$. Then we consider an $m \times m$ matrix $\boldsymbol{Q}$ expressed by

$$
\boldsymbol{Q}=\sum_{i=1}^{m} \boldsymbol{C}_{\boldsymbol{y}, i}^{(4)}
$$

It is shown by a simple calculation (see [6]) that (17) becomes

$$
\boldsymbol{Q}=\boldsymbol{H} \tilde{\boldsymbol{\Lambda}} \boldsymbol{H}^{T},
$$

where $\tilde{\Lambda}$ is a diagonal matrix defined by

$$
\begin{aligned}
& \tilde{\Lambda}:=\operatorname{diag}\left\{\gamma_{1} \tilde{a}_{1}, \gamma_{2} \tilde{a}_{2}, \cdots, \gamma_{n} \tilde{a}_{n}\right\} \\
& \tilde{a}_{r}:=\sum_{i=1}^{m} h_{i r}^{2}, \quad r=1,2, \cdots, n,
\end{aligned}
$$

and $\operatorname{diag}\{\cdots\}$ denotes a diagonal matrix with the diagonal elements built from its arguments.

Here, as a constraint, we take the following value:

$$
\begin{aligned}
\left|C_{z y}\right| & =\left|\sum_{i=1}^{m} \operatorname{cum}\left\{z_{l}(t), z_{l}(t), y_{i}(t), y_{i}(t)\right\}\right|=\left|\boldsymbol{w}_{l}^{T} \boldsymbol{Q} \boldsymbol{w}_{l}\right| \\
& =\left|\sum_{i=1}^{n} \tilde{a}_{i} \gamma_{i} g_{l i}^{2}\right|
\end{aligned}
$$

Then, we consider of solving the problem that the fourth-order cumulant $\left|C_{z x}\right|$ is maximized under the condition that $\left|C_{z y}\right|=$ $\left|\tilde{a}_{\rho_{l}} \gamma_{\rho_{l}}\right|$. By the Lagrangian method, the following generalized eigenvector problem is derived from the problem:

$$
\boldsymbol{C}_{y x} \boldsymbol{w}_{l}=\tilde{\lambda} \boldsymbol{Q} \boldsymbol{w}_{l}
$$

From the following theorem, one can see that by solving the eigenvector problem of the matrix $\boldsymbol{Q}^{\dagger} \boldsymbol{C}_{y x}$, its solutions, that is, the $n$ eigenvectors $\boldsymbol{w}_{l}(l=1,2, \cdots, n)$ correspond to the vectors $\tilde{\boldsymbol{w}}_{l}(l=1,2, \cdots, n)$ in (4).

Theorem 2: Suppose the values $a_{i}^{2} / \tilde{a}_{i}, i=1,2, \cdots, n$ are all nonzero and distinct. Then the $n$ eigenvectors corresponding to $n$ nonzero eigenvalues of $\boldsymbol{Q}^{\dagger} \boldsymbol{C}_{y x}$ become the vectors $\tilde{\boldsymbol{w}}_{l}$, $l=1,2, \cdots, n$, satisfying (4).

Proof: Based on (22), we consider the following eigenvector problem:

$$
\boldsymbol{Q}^{\dagger} \boldsymbol{C}_{y x} \boldsymbol{w}_{l}=\lambda \boldsymbol{w}_{l} .
$$

Then, from (11), (18), and the property of the pseudo-inverse operation, (23) becomes

$$
\boldsymbol{H}^{T \dagger} \tilde{\boldsymbol{\Lambda}}^{-1} \boldsymbol{\Lambda} \boldsymbol{H}^{T} \boldsymbol{w}_{l}=\lambda \boldsymbol{w}_{l} .
$$

Multiplying (24) by $\boldsymbol{H}^{T}$ from left side and using a property of the pseudo-inverse operation again, (24) becomes

$$
\tilde{\boldsymbol{\Lambda}}^{-1} \boldsymbol{\Lambda} \boldsymbol{H}^{T} \boldsymbol{w}_{l}=\lambda \boldsymbol{H}^{T} \boldsymbol{w}_{l}
$$

By noting that $\tilde{\boldsymbol{\Lambda}}^{-1} \boldsymbol{\Lambda}$ is a diagonal matrix whose elements, $a_{i}^{2} / \tilde{a}_{i}, i=1,2, \cdots, n$, are all nonzero and distinct, if $\boldsymbol{g}_{l}:=$ $\boldsymbol{H}^{T} \boldsymbol{w}_{l} \neq 0$, then the eigenvectors $\boldsymbol{g}_{l}$ obtained from (25) become the vectors $\tilde{\boldsymbol{g}}_{l}$ satisfying (4). Namely, the $n$ eigenvectors $\boldsymbol{w}_{l}$ corresponding to $n$ nonzero eigenvalues of $\boldsymbol{Q}^{\dagger} \boldsymbol{C}_{y x}$ obtained from (23) become the vectors $\tilde{\boldsymbol{w}}_{l}, l=1,2, \cdots, n$, satisfying (4).
Remark 2: Since the matrix $\boldsymbol{Q}^{\dagger} \boldsymbol{C}_{y x}$ consists of only fourthorder cumulants, the eigenvectors derived from the matrix can be obtained with as little influence of Gaussian noise as possible, which is referred as a robust eigenvector algorithm (REVA). From the matrix $\tilde{\Lambda}^{-1} \boldsymbol{\Lambda}$ in (25), it can be seen that the fourth-order cumulants $\gamma_{i}$ are canceled each other. Therefore, the eigenvector algorithms (22) can be applied to the case that the signs of the fourth-order cumulants $\gamma_{i}(i=1,2, \cdots, n)$ are different, that is, sub-Gaussian and super-Gaussian signals are treated as source signals.

Remark 3: The proposed EVAs in (7) and (22), both are closely related to the joint diagonalization (e.g., [1], [3]).

\section{Discussion}

In this section, let us consider the case that as a reference signal,

$$
x(t)=\boldsymbol{w}_{l}^{T}(t-1) \boldsymbol{y}(t):=\boldsymbol{g}_{l}^{T}(t-1) \boldsymbol{s}(t)
$$

is used, where $\boldsymbol{w}_{l}^{T}(t-1)$ denotes the vector obtained by the proposed EVAs at time $t-1$ and $\boldsymbol{g}_{l}^{T}(t-1)=\left[g_{l 1}(t-1), g_{l 2}(t-\right.$ $\left.1), \cdots, g_{l n}(t-1)\right]:=\boldsymbol{w}_{l}^{T}(t-1) \boldsymbol{H}$. Then, the vector $\boldsymbol{w}_{l}(t)$ obtained by the proposed EVAs is calculated by using the previous eigenvector $\boldsymbol{w}_{l}(t-1)$. This means that the proposed EVAs are iteratively carried out. Under the reference signal $x(t)$ in (26), the matrix $\boldsymbol{C}_{y x}$ can be expressed as

$$
\boldsymbol{C}_{y x}=\boldsymbol{H} \dot{\mathbf{\Lambda}} \boldsymbol{H}^{T},
$$

where $\dot{\Lambda}$ is a diagonal matrix with the diagonal elements $g_{l i}^{2}(t-1) \gamma_{i}, i=1,2, \cdots, n$.

Since the diagonal elements of $\dot{\boldsymbol{\Lambda}}$ include $g_{l i}, i=1,2, \cdots, n$, which are the elements of the vector $\boldsymbol{g}_{l}$, if the vector $\tilde{\boldsymbol{w}}_{l}$ satisfying (4) is obtained by the proposed EVAs with (26), the diagonal elements of $\dot{\boldsymbol{\Lambda}}$ become zero except for $\rho_{l}$ th diagonal element, that is, $\dot{\boldsymbol{\Lambda}}=\operatorname{diag}\left\{0, \cdots, 0, g_{l \rho_{l}}^{2}(t-1) \gamma_{\rho_{l}}\right.$ ( $\rho_{l}$ th element), $\left.0, \cdots, 0\right\}$. This means that if the proposed EVAs with the reference signal (26) are iteratively used to estimate the vector $\tilde{\boldsymbol{w}}_{l}$ in (4), only one source signal can be separated from $\boldsymbol{y}(t)$. This ability is the same as the well-known method obtained by the constrained maximization problem [8]. In order to show different abilities from the conventional methods which have such an ability, therefore, at least, one needs to choose reference signals such that the diagonal elements of $\boldsymbol{\Lambda}$ in (11) are of fixed values.

\section{COMPUTER SIMUlations}

To demonstrate the validity of the proposed methods, many computer simulations were conducted. Some results are shown in this section. The unknown system $\boldsymbol{H}$ was set to be a $4 \times$ 3 matrix, that is, a three-input four-output system:

$$
\boldsymbol{H}=\left[\begin{array}{ccc}
1.0 & 0.4 & 0.6 \\
0.7 & 1.0 & -0.3 \\
0.2 & -0.5 & 1.0 \\
-0.45 & 0.25 & 0.7
\end{array}\right]
$$

The three inputs $s_{i}(t)(i=1,2,3)$ of the system $H$ were two sub-Gaussian signals and one super-Gaussian signal, in which each sub-Gaussian signal takes one of two values, 


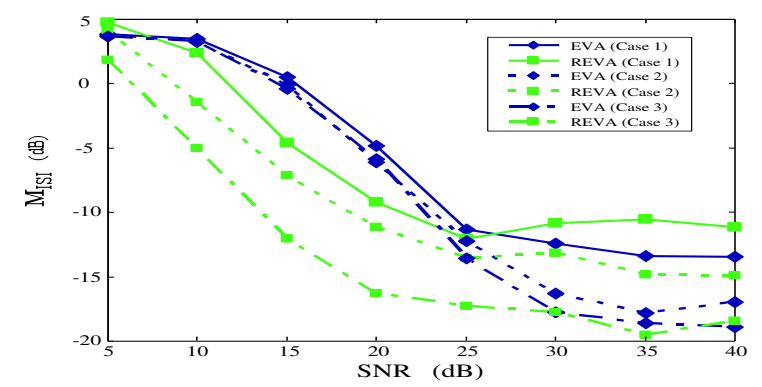

Fig. 2. The average performances of the REVA and the EVA with varying the SNR level, in the three cases of (i) 5000 samples, (ii) 10000 samples, (iii) 30000 samples.

-1 and 1 with equal probability $1 / 2$ and the super-Gaussian signal takes $-2,2$, and 0 with probabilities $1 / 8,1 / 8$, and $6 / 8$, respectively. The filter $\boldsymbol{f}$ making a reference signal was set to be $\boldsymbol{f}=[0,1,0,0]^{T}$. The Gaussian noises $n_{i}(t)(i=1,2,3,4)$ with their variances $\sigma_{n_{i}}^{2}$ were included in the outputs $y_{i}(t)$ at various SNR levels. The SNR was considered at the output of the system $\boldsymbol{H}$. As a measure of performance, we used the multichannel intersymbol interference $\left(\mathrm{M}_{\mathrm{ISI}}\right)$ defined in [6].

Fig. 2 shows the performances of the proposed EVA and REVA in the cases where the SNR levels were taken from $5 \mathrm{~dB}$ to $40 \mathrm{~dB}$, in which each $\mathrm{M}_{\text {ISI }}$ shown in Fig. 2 was the average of the performance results obtained by 50 independent Monte Carlo runs. In each Monte Carlo run, the matrices $\boldsymbol{R}$, $\boldsymbol{Q}$, and $\boldsymbol{C}_{y x}$ were estimated by data samples in the following three cases: (Case 1) 2,500 data, (Case 2) 5,000 data, and (Case 3) 10,000 data. Note that the matrices $\boldsymbol{R}, \boldsymbol{Q}$, and $\boldsymbol{C}_{y x}$ were estimated by using the following on-line methods, in which the matrix $\boldsymbol{R}$ was estimated by $\tilde{\boldsymbol{V}}_{1}(t)$ in (30).

$$
\begin{aligned}
& \boldsymbol{Q}(t):=\beta_{1} \boldsymbol{Q}(t-1)+\left(1-\beta_{1}\right)\left\{\boldsymbol{V}_{1}(t) \boldsymbol{V}_{1}^{T}(t)\right. \\
& \left.-2 \boldsymbol{V}_{1}(t) \tilde{\boldsymbol{V}}_{1}^{T}(t)-\operatorname{tr}\left\{\tilde{\boldsymbol{V}}_{1}(t)\right\} \boldsymbol{V}_{1}(t)\right\},
\end{aligned}
$$

where $\boldsymbol{V}_{1}(t):=\boldsymbol{y}(t) \boldsymbol{y}^{T}(t), \operatorname{tr}\{X\}$ denotes the trace of the matrix $X$, and $\tilde{\boldsymbol{V}}_{1}(t)$ is a moving average of $\boldsymbol{V}_{1}(t)$ calculated by

$$
\tilde{\boldsymbol{V}}_{1}(t)=\beta_{2} \tilde{\boldsymbol{V}}_{1}(t-1)+\left(1-\beta_{2}\right) \boldsymbol{V}_{1}(t), .
$$

The vector $\boldsymbol{C}_{y x}$ in (22) was calculated using a moving average defined by

$$
\begin{array}{r}
\boldsymbol{C}_{y x}(t):=\beta_{1} \boldsymbol{C}_{y x}(t-1)+\left(1-\beta_{1}\right)\left\{x^{2}(t) \boldsymbol{y}(t) \boldsymbol{y}^{T}(t)\right. \\
\left.-2 \tilde{v}_{x 1}(t) x(t) \boldsymbol{y}^{T}(t)-\tilde{v}_{x 2}(t) \boldsymbol{y}(t) \boldsymbol{y}^{T}(t)\right\},
\end{array}
$$

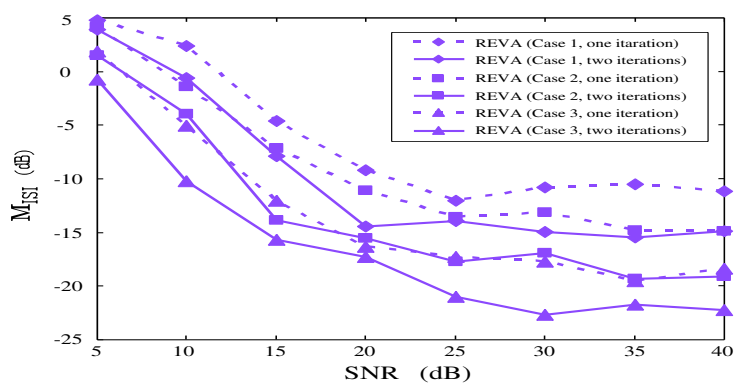

Fig. 3. The comparison results of the performances of the RSEM and the SEM, obtained by varying the SNR level and different iterations. where $\tilde{v}_{x i}(t)$ is a moving average defined by

$$
\tilde{v}_{x i}(t)=\beta_{2} \tilde{v}_{x i}(t-1)+\left(1-\beta_{2}\right) v_{x i}(t), \quad i=1,2,
$$

where $v_{x 1}(t)=x(t) \boldsymbol{y}(t)$ and $v_{x 2}(t)=x^{2}(t)$. The parameters $\beta_{1}$ and $\beta_{2}$ were set to be 0.99999 and 0.995 , respectively.

It can be seen from Fig. 2 that for the case that the SNR level is more than $25 \mathrm{~dB}$, the EVA is more useful than the REVA, because the EVA can provide better performances than the REVA using a few data samples. On the other hand, the REVA is effective for the case that the SNR level is less than $25 \mathrm{~dB}$, but the performance of the REVA depends on the accuracy of the estimate of the matrix $\boldsymbol{Q}$. Indeed, the results (solid lines shown in Fig. 3) obtained by two iterations using the above on-line estimation methods of $\boldsymbol{Q}$ and $\boldsymbol{C}_{y x}$ were better than those shown in Fig. 2 (see doted lines shown in Fig. 3), in which for each iteration, to implement (29) and (32), the data samples in the above three cases were used. Here, the first iteration started with appropriate initial values, and the second iteration started with the initial values obtained from the first iteration.

From all the results, we conclude that the EVA is effective for the case of a little influence of Gaussian noise and the REVA is useful for the case that Gaussian noise level is high, but it depends on the accuracy of the estimate of the fourthorder cumulant matrix $\boldsymbol{Q}$.

\section{CONCLUSIONS}

We have proposed two kinds of EVAs for solving the BSS problem. By using reference signals, these are capable for separating source signals simultaneously from their mixtures. One of the EVAs is robust against Gaussian noise, which means that the EVA can be used to estimate the (pseudo-) inverse of $\boldsymbol{H}$ with as little influence of Gaussian noise as possible. Computer simulations have demonstrated the validity of the proposed EVAs.

\section{REFERENCES}

[1] A. Adib, E. Moreau, and B. Aboutajdine, "Blind source separation by simultaneous generalized referenced contrasts diagonalization," Proc. ICA2003, pp. 657-661, Nara, Japan 2003.

[2] A. Adib, E. Moreau, and D. Aboutajdine, "Source separation contrasts using a reference signal," IEEE Signal Processing Letters, Vol. 11, No. 3, pp. 312-315, March 2004.

[3] J.-F. Cardoso and A. Souloumiac, "Jacobi angles for simultaneous diagonalization," SIAM J. Matrix Anal. Appl., Vol. 17, pp. 161-164, 1996.

[4] B. Jelonnek and K. D. Kammeyer, "A closed-form solution to blind equalization," Signal Processing, 36(3), pp. 251-259, 1994.

[5] B. Jelonnek, D. Boss, and K. D. Kammeyer, "Generalized eigenvector algorithm for blind equalization," Signal Processing, 61(3), pp. 237-264, 1997.

[6] M. Kawamoto, K. Kohno, and Y. Inouye, "Robust Super-Exponential Methods for Deflationary Blind Source Separation of Instantaneous Mixtures," IEEE Transactions on Signal Processing, Vol. 53, No. 5, pp. 1933-1937, May 2005.

[7] P. Lancaster and M. Tismenetsky, The Theory of Matrices, second edition, Academic Press, INC., 1985.

[8] O. Shalvi and E. Weinstein, "New criteria for blind deconvolution of nonminimum phase system (channels)," IEEE Trans. Inform. Theory, vol. 36, no. 2, pp. 312-321, 1990.

[9] L. Tong, Y. Inouye, and R.-w. Liu, "Waveform-preserving blind estimation of multiple independent sources," IEEE Trans. Signal Processing, vol. 41, no. 7, pp. 2461-2470, Jul. 1993 